\title{
Du sollst kein falsches Zeugnis geben
}

\author{
B. Gurtner
}

Im Ärztestand gibt es prozentual wahrscheinlich ebenso viele notorische Lügner, gelegentliche Schummler und grundehrliche Menschen wie in der Gesamtbevölkerung. Der Beruf bringt es aber mit sich, dass wir immer wieder in Situationen geraten, in denen wir uns versucht oder gar gezwungen fühlen, die Wahrheit etwas zurechtzubiegen. Schon während des Medizinstudiums erfuhren wir bei Praxisvertretungen, dass Pockenimpfzeugnisse da und dort erhältlich waren, ohne dass die überängstlichen Kunden einen Kratzer erlitten hatten. Arbeitsunfähigkeit wurde unter zwingenden Umständen doppelblind bestätigt. Die präsenile Fahrtüchtigkeit liess sich problemlos bescheinigen, weil eine rasch hingekritzelte Unterschrift viele Diskussionen und Verärgerungen ersparte. Als die Fristenlösung noch in ferner Zukunft lag, wurden auch Schwangerschaftsabbrüche mit fragwürdigen Gutachten legalisiert.

Der Stempel «militärdienstuntauglich» war bei einigen ideologisch vorgespurten Kollegen auch von durchtrainierten Drückebergern leicht zu bekommen. Truppenärzte dispensierten Bergbauern wegen «Rückenleidens» vom Wiederholungskurs, wenn der WK ungünstigerweise in die Zeit der Heuernte fiel. Sozial gesinnte Hausärzte setzten den Invaliditätsgrad arbeitsloser Familienväter möglichst hoch an und verteidigten den Prozentsatz gegenüber der IV-Kommission. Ist die Annahme irrig, dass diese Praktiken auch heute noch gelegentlich üblich sind? Wie wirkt sich wohlmeinendes Mogeln auf das Arzt-Patienten-Verhältnis aus? Verbleiben Bewunderung für die Zivilcourage, Dankbarkeit oder eben doch leichtes Misstrauen, weil sich der Arzt als nicht ganz ehrlich erwiesen hat?

Praxisinhaber oder Klinikleiter verwenden in Arbeitszeugnissen vieldeutige Formulierungen, um weder der Wahrheit noch den Qualifizierten allzuviel Leid anzutun. Ein oft nörgelnder Mitarbeiter, der wegen gemeiner Intrigen entlassen werden musste, war dennoch mit der Feststellung «er hat von dem allen zustehenden Recht auf freie Meinungsäusserung ausgiebig Gebrauch gemacht» nicht zufrieden. Bei anstehenden Chefarztwahlen mussten überschwengliche Empfehlungsschreiben darauf abgeklopft werden, ob nicht der unterzeichnende Klinikdirektor einen unfähigen Oberarzt wegzubefördern versuchte. Sonst kam es vor, dass leitende Ärzte aus Universitätskliniken zu leidenden Angestellten an Landspitälern wurden, wo man sich bald wieder von ihnen trennen musste.

Wie in letzter Zeit mehrmals zu erfahren war, wurden unerwünschte Tatsachen auch in einigen Studienberichten verfälscht oder unterdrückt, sehr zum Schaden vieler Patienten, denen die hochgejubelten Medikamente verkauft worden sind. Systematisch angepasst wird die Wahrheit, sobald Diagnosen oder Codes finanzielle Auswirkungen haben. Bei der gestaffelten Einführung der DRG (diagnosis-related groups) in den amerikanischen Bundesstaaten verschlechterte sich Gesundheitsstatistik sprunghaft in der gleichen Reihenfolge, weil nach dem Systemwechsel viele leichte Erkrankungen zu einträglicheren schweren Leiden deklariert wurden. Dieses Miscoding brachte auch die Gesundheitsreform in Oregon zu Fall, die das Ziel hatte, dank eingeschränkter Grundleistungen die Mittel für einen Versicherungsschutz aller Einwohner frei zu machen. Eingriffe oder Medikamente, die nicht mehr in der Liste enthalten waren, wurden mit entschädigungsberechtigten Positionen getarnt, Eigennutz kam vor dem Gemeinwohl. Auch bei uns scheinen einige ICD-10-Profis die Kunst des Upgradings zu kennen, der lockere Umgang mit Diagnosen folgt dem kampflosen Verzicht auf das Arztgeheimnis und macht uns alle unglaubwürdig.

Ob man den Kranken schlechte Befunde offenlegen muss oder darf, lässt sich nicht in eine allgemeingültige Empfehlung fassen. Kulturell bedingt liegen die Meinungen, immer allen alles zu sagen oder bis zum letzten Atemzug zu vertuschen, weit auseinander. Sie werden zur unbarmherzigen Doktrin, wenn nicht das Bedürfnis der Kranken, sondern der voreingenommene Standpunkt der Helfer entscheidend ist (Paul Sporken). Das - soweit abschätzbar schonendste Vorgehen muss jedesmal der Situation angepasst werden. Man wird die bittere Wahrheit nicht aufdrängen, sollte aber das, wonach gefragt wird, nie mit einer blanken Lüge zudecken, die zu oft durchschaut wird. Wenn die Betreuenden kein noch so gutgemeintes «falsches Zeugnis» abgeben und stets einen Funken Hoffnung belassen, werden ihnen die Kranken und ihre Angehörigen auch in schwierigen Zeiten eher das volle Vertrauen schenken. 